\title{
Toward a Discourse Structure Account of Speech and Attitude Reports
}

\author{
Antoine Venant ${ }^{1,2}$ \\ 1 Laboratoire Parole et Langage \\ 2 Ecole Normale Superieure de Cachan
}

\begin{abstract}
This paper addresses the question of propositional attitude reports within Segmented Discourse Representation Theory (SDRT). In line with most SDRT discussions on attitudes reports, we argue that the reported attitude should be segmented in the same way as the rest of the discourse is. We identify several issues that are raised by the segmentation of attitude reports. First, the nature of some relations crossing the boundaries between main and embedded speech remains unclear. Moreover, such constructions are introducing a conflict between SDRT's Right Frontier Constraint (RFC) and well established facts about accessibility from factual to modal contexts. We propose two solutions for adapting discourse structure to overcome these conflicts. The first one introduces a new ingredient in the theory while the second one is more conservative and relies on continuation-style semantics for SDRT.
\end{abstract}

\section{Introduction}

From a semantic perspective, attitudes reports require to solve several notorious puzzles. Among these, are a lot of problems triggered by definites: Substitution of directly co-referential expressions is generally not allowed under the scope of an attitude verb and neither does existential generalization(see the shortest spy problem raised by [8]). Closely related to those are effects of attitudes verbs on discourse referents availability. For instance factive epistemic verbs like 'to know' allow referents introduced under their scope to be then referred from outside their scope, while non factive like 'to believe' do not. These two issues are related to context which has naturally led to several accounts involving dynamic semantics such as $[1,9]$.

From the modeling text coherence perspective, we need to understand how reporting someone's propositional attitude interacts with the overall discourse structure. The dynamic framework of Segmented Discourse Representation Theory (SDRT) [3] allows to address both perspectives simultaneously by looking at the interaction between discourse structure and anaphoric phenomena. However there is in SDRT no semantic contribution for attitudes report that is as precise as the ones cited above and formulated within Discourse Representation Theory (DRT) [7]. Since SDRT builds over a lower-level formalism ( $D R T)$, and enriches it by adding rhetorical relations, one may wonder whether DRT-style accounts could be straightforwardly embedded in SDRT. One condition for this is that SDRT keeps the benefits of the work done in the chosen low-level logic, and uses its ability to handle discourse relation to model a more

R.K. Rendsvig and S. Katrenko (Eds.): ESSLLI 2012 Student Session Proceedings, CEUR Workshop Proceedings vol.: see http://ceur-ws.org/, 2012, pp. 170-179. 
accurate interface between semantics and pragmatics. ${ }^{3}$ We want to address then the question of how does SDRT's treatment of embedded speech acts keeps up with such a consideration.

We attach a particular attention to examples in the spirit of 1 for they involve irruption of the factive context into the modal context at the discourse level. We think that such anaphoric links are not fully modelled by the current analyses of attitude reports in SDRT. Distinguishing between Intentional/Evidential uses of reportative verbs still do not allow them in some intensional cases while DRT based approaches would very likely allow event correference from an embedded DRS to the main DRS.

Example 1. The criminal parked his car somewhere near the airport. So detectives think that afterwards he tried to get into a plane.

After briefly introducing SDRT in section 2, we argue in section 3 for segmentation of reported constructions. Section 4 deals with relations that links a reported speech act to a factual one. It shows that the discursive structure of intensional reports is closed to incoming relations, but still can bear anaphoric links to the context. On this basis it exhibits a family of relations for which RFC makes bad predictions. Section 5 presents two ways of restoring the right accessibility conditions while still benefiting from SDRT specificities.

\section{Segmented Discourse Representation Structures}

SDRT assumes that to analyze discourse one has to segment into meaningful units that shall be linked to each other by means of discourse relations. Each segment is called an elementary discourse unit (edu). The level of segmentation is merely the clause level (where a clause can be understood as something containing an event or a state) ${ }^{4}$.

Each discourse unit is assigned a label $\left(\pi_{i}, \ldots \pi_{n}\right)$ in the language of SDRSs and a corresponding formula in a given language for representation of atomic clauses $\left(K_{1}, \ldots, K_{n}\right)^{5}$. These labels will serve as arguments of rhetorical relations, like narration $\left(\pi_{i}, \pi_{j}\right)$ or explanation $\left(\pi_{i}, \pi_{j}\right)$. Additional labels are associated with complex structured content made of rhetorical relations and other subordinated labels. Such labels with complex content will be called complex discourse units and recursively used as argument of other relations. A SDRS is a triple $\langle A, \mathcal{F}$, Last $\rangle$ where $A$ is a set of labels (A), $\mathcal{F}$ a function mapping labels to contents (either lower-level language such as DRS or discourse relations in case of complex constituents) and Last the information of the last segment introduced. (See [3]:p.138 for the precise definition).

SDRT makes a structural distinction between coordinating and subordinating relations. The former, like narration, confer an equal status to their two arguments. The latter introduce a hierarchy between the related constituents. Such a distinction allows to define the so-called Right Frontier constraint. The Right Frontier is the set of labels $R F=\left\{\pi \mid \pi \prec^{*}\right.$ Last $\}$ where $\prec^{*}$ is the transitive closure of the dominance relation $\prec$ defined by $\pi \prec \alpha$ iff $\alpha$ is a complex consitutent which immediately outscopes $\pi$ or

\footnotetext{
${ }^{3}$ This is indeed what the theory aims at doing while extending DRT's definition of accessibility.

${ }^{4}$ How fine-grained segmentation should is still under discussions. The present work is also a contribution at this level since we argue for segmenting attitudes.

${ }^{5}$ This is the lower-level language and associated representations.
} 
there is subordinating edge $R(\alpha, \pi)$ in some constituent $\gamma$. The Right Frontier Constraint stipulates that labels accessible for discourse continuation are those of the Right Frontier, while the ones accessible for correference have to be DRS-accessible on the right frontier.

For instance, the structure of [John visited his friend $]_{a}$. [Then he went to the cinema $]_{b}$. [He watched Pirates of the Caribbean $]_{c}$ is narration $\left(\pi_{a}\right.$, elaboration $\left.\left(\pi_{b}, \pi_{c}\right)\right)$ elaboration is a subordinating and narration a coordinating relation, therefore the right frontier is $\left\{\pi_{b}, \pi_{c}\right\}$ and the discourse could not be felicitously continued by They talked for a long time which intends to attach to $a$.

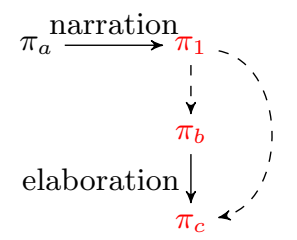

Fig. 1. SDRS example

Figure 1 gives a graphical representation of this example, with the convention that coordinating relation are drawn horrizontally, subordinating one vertically, complex constituents are linked with dashed edges to their subconstituents, and nodes of the right frontier are red.

The truth conditional content of an SDRS, as in DRT, is expressed in terms of context-change potential (i.e relation between world-assignment pairs), and is recursively computed using the semantic constraints associated with each rhetorical relation, finally relying on the lower-level logical forms.

In this framework, we now move to the discourse structure of attitude and speech reports.

\section{Segmentation and treatment of the matrix clause}

There are at least two reasons for capturing the interaction between attitudes or speech reports and discourse structure. First, we need to account for discourse phenomena both inside the reports and across their boundaries. Then the treatment of intentional and evidential uses of attitude reports in the way of [6] also require segmentation.

About the first point, example 2 is not felicitous, because the pronoun ' $i t$ ' cannot easily refer to the salmon in the given context. Such a behaviour is predicted by RFC. Therefore, even if the semantics of attitudes generally involves quantification over intensions or contents, and thus erases to some extent the structure of the logical form of the original speech act, the discourse structure of the report is needed anyway to build the logical form of the speech report.

Example 2. \#John told me that Marry had a wonderful evening last night. He said [she ate salmon $]_{a}$ [and then won a dancing competition $_{b}$ ] [and that it was beautiful pink. $]_{b}$ 
On the other hand, in example 3 the reported speech introduces a narration between two events while the non-reported discourse asserts a causal relation (result) between the two same events. The contrast introduced by but is however coherent, partially because it is supported by the isomorphic structures of the reported speech and the non-reported one. SDRT treatment of contrast as a scalar relation, following $[2,3]$ provides such an analysis, assuming that the structure of the embedded speech is accessible.

Example 3. John says that he left after Mary did but he left because she did.

About the segmentation of the matrix, we may consider the matrix clause as nothing more than a kind of logical operator ${ }^{6}$. However, that would be inaccurate since the matrix clause can be fairly sophisticated. It generally includes a communication event or a mental state that can be modified by adverbs or prepositional phrases and therefore would be difficult to model as simple logical operator. Since removing the matrix-clause from the discourse representation is not an option neither segmenting attitude reports forces us to deal with this matrix-clause segment.

[6] addresses several issues raised by such a treatment of reported speech. The approach consists in segmenting apart matrix clause and reported speech and in identifying the relation between these elements themselves but also their relations with the surrounding context. It distinguishes between two uses of reportative verbs, namely evidential where the embedded content is asserted by the main speaker and intensional where the content of the report is not asserted by the main speaker. In evidential uses, the matrix clause is subordinated to the embedded content by a veridical evidence relation. ${ }^{7}$ In intensional uses, the embedded content is subordinated to the matrix via a relation of attribution which is non-veridical. Such a distinction makes very profitable the separation of the matrix clause and the reported speech, accounting for cases like 4 .

Example 4. (1) [The neighbours are gone. $]_{a}[\text { John told me that }]_{b}$ [they went on vacation in an expensive hotel. $]_{c}$ [I called it this morning. $]_{d}$

(2) [The neighbours are gone. $]_{a}[\text { John told me that }]_{b}$ [they went on vacation in an expensive hotel. $]_{c}[\text { But he lied }]_{e}$.

As [6] argues, we can see in the first example above that $c$ is asserted by the speaker since $d$ is carrying an anaphoric link to the hotel eventhough it has first been introduced under the scope of the attitude ${ }^{8}$. On the contrary, in 4.2 , the author disagree with what is reported, and the existence of the hotel is not ensured anywhere outside the scope of the attitude. Therefore The hotel shall not be referred to later in the discourse. [6] also argues that the compositional semantics of both the reported speech and the matrix clause do not change from an intensional to an evidential report. And the matrix clause can neither be deleted without loss of compositional content in the one nor the other case. But the way the two parts of speech are related can change. Fu rthermore, since

${ }^{6}$ This would still requires to modify the SDRT framework since all logical operators are delegated either to the lower-level logical forms or to the semantic effects of discourse relations.

${ }^{7}$ To be satisfied, veridical relations require their arguments to be true in the model. Non veridical relations do not have this requirement. [3]

${ }^{8}$ At least if we assume that $d$ is not part of what John said here, but in that case that it would be a very odd reading. 
the two first sentences are the same in both examples, the decision of choosing the one or the other might only be a matter of context, as such it is essentially information packaging, and in SDRT, this level is kept aside from the logic of information content.

Following this analysis, in the first example, $d$ will be related to $c$ by a veridical relation of narration, forcing the evidential reading. So $c$ will be related to $b$ with the veridical Evidence $(c, b)$ and to $a$ with a veridical relation of explanation. In the second example however, continuation $e$ is attached to the whole report with a contrast and yields an intensional reading (attaching $e$ to the embedded clause only would entails that John said something incoherent, which is less likely the intended meaning) and $b$ is related to $c$ using the non-veridical attribution $(b, c)$. The two different type of structures are sketched below. (Left column is evidential, right one is intensional. We also give some of the semantics conditions associated with the two relations involved).

$$
\begin{aligned}
& \mathcal{F}\left(\pi_{b}\right)=\frac{\phi}{A(x, \phi)} \quad \mathcal{F}^{\prime}\left(\pi_{b}\right)=\frac{\phi}{A(x, \phi)} \\
& \mathcal{F}\left(\pi_{\text {top }}\right)=R_{e}\left(\pi_{a}, \pi_{c}\right) \wedge \text { evidence }\left(\pi_{c}, \pi_{b}\right) \mathcal{F}^{\prime}\left(\pi_{\text {top }}^{\prime}\right)=R_{i}\left(\pi_{a}, \pi_{b}\right) \wedge \operatorname{attribution}\left(\pi_{b}, \pi_{c}\right) \\
& \Phi_{\text {evidence }\left(\pi_{c}, \pi_{b}\right)} \Rightarrow K_{\pi_{c}} \wedge K_{\pi_{b}} \wedge \phi \sim{ }^{\wedge} \pi_{c} \Phi_{\text {attribution }\left(\pi_{b}, \pi_{c}\right)} \Rightarrow K_{\pi_{b}} \wedge \phi \sim^{\wedge} \pi_{c}
\end{aligned}
$$

Where $\sim$ may be understood as an equivalence relation between SDRS contents. How this content and $\sim$ are defined actually remains an open question. Basically content could be understood as the context change potential. However, blocking substitution of logically equivalent expressions under the scope of an attitude verb may require some amount of structure being kept in the notion of content([1]).

\section{Relations across boundaries}

As [5] remarks, the picture becomes more complicated when relations comes to cross the boundaries of an embedded speech act such as in 5 .

Example 5. [Fred will go to Dax for Christmas $]_{a}$. [Jane claims that $]_{b}[$ Afterwards, he will go to $\mathrm{Pau}]_{c}$.

Afterwards introduces a veridical relation of narration. If we invoke the evidential/intensional distinction and assume an evidential reading, this example does not pose any problem since the discourse producer $(D P)$ is thought to assert the content $\pi_{c}$ and thus can use a veridical relation for relating it to the context. However, with an intensional reading the speaker does not claim narration $\left(\pi_{a}, \pi_{c}\right)$ since he does not assert the content of $\pi_{c}$. But he still can commit to Jane committing to such a relation. To solve this problem, [5] set up a new paradigm for discourse analysis that examines reported relations against several sources. For instance, 5 will be analyzed as follows:

The discourse producer is certain of the main eventuallity $e_{a}$ in $a$ but he does not know anything about the one in $c$. Jane is attributed to be certain about the main eventuality in $c$, and, after the source of the narration is identified to being Jane, the picture is completed with the statements of Jane being certain of $e_{a}$ too, as well as $e_{a}$ and $e_{c}$ being in a temporal sequence. Semantically speaking, such examples require some further discussion. First, we cannot always identify a source for a relation. Consider a two level deep embedding as in 6 . Asserting narration Fred's wife $\left(\pi_{a}, \pi_{d}\right)$ in this case would make us unable to distinguish between 6 and the same without $b$. With 6 the writer does not commit to Fred's wife committing that he will go to Pau. 
Example 6. [Fred will go to Dax for Christmas $]_{a}$. [Jane told me that $]_{b}$ [according to his wife, $]_{c}$ [afterwards, he will go to Pau $]_{d}$.

Besides, interpreting narration ${ }_{J}\left(\pi_{a}, \pi_{c}\right) \wedge$ attribution $\left(\pi_{b}, \pi_{c}\right)$ requires some precisions that the framework does not provide. To this end, we may switch to a dialogical framework in which each individual would receive its own SDRS. However, reducing reported speech and other voicing effects to dialogue is not what we want to do (especially if we want to be able to account for 6 ). Another way to provide an interpretation would be to use [10] semantics, $R(a, b): \llbracket C\left(\operatorname{Speaker}(b), K_{a}\right) \wedge C\left(\operatorname{Speaker}(b), K_{b}\right) \wedge$ $C\left(\right.$ Speaker $\left.(b), \phi_{R(a, b)}\right) \rrbracket$ where $C$ is a commitment relation and $R$ a veridical relation. But once again the structure is misleading, and with this account, the narration producer must be understood as being Jane, which is strange since even when being reported, its producer remains the main producer. And we would end up with a fomulla entailing $C\left(\right.$ Jane, $\left.\phi_{\text {narration }\left(\pi_{a}, \pi_{b}\right)}\right)$ instead of something like $C\left(W, C\left(\right.\right.$ Jane, $\left.\left.\phi_{\text {narration }\left(\pi_{a}, \pi_{b}\right)}\right)\right)$ that would be needed to account for example 6 with this semantics. Our conclusion thus is that the problem originates from the structure which does not model the right scope of attribution which should includes the narration relation. This can be done in SDRT by introducing a complex segment for representing the embedded content ${ }^{9}$ :

$$
\begin{gathered}
A=\left\{\pi_{\text {top }}, \pi_{a}, \pi_{b}, \gamma, \pi_{c}\right\} \\
\mathcal{F}\left(\pi_{\text {top }}\right)=\operatorname{attribution}\left(\pi_{b}, \gamma\right) \mathcal{F}(\gamma)=\operatorname{narration}\left(\pi_{a}, \pi_{c}\right)
\end{gathered}
$$

Equation 1 is actually missing a non-embedded left-veridical coherence relation between $\pi_{a}$ and another segment. As it stands our structure semantics does not imply that the main discourse producer claims the content of $a$. However, more generally there must be some relation $(R)$ introduced by the main producer and that links $\pi_{a}$ with the speech act of reporting Jane's claim (at least with an intensional reading). Attribution being subordinating in the intensional case, $R$ cannot be coordinating without the RFC being violated in 5 . So it seems that $R$ should be a subordinating like background. However cases like 7 are source of problems.

Example 7. (1) [The train arrived 3 hours late.] [then the company announced that] [in consequence, the passengers would be refunded]. [But as a matter of fact, they never were.]

(2) [John had a deadline at midnight yesterday.] [So we all though that afterwards he would go to bed.] [But he did not.]

(3) [Yesterday, John fell three times in a row.] [Mary then told him that] [it was probably because he drank to much.] [He did not believe her.]

All these examples involve an intensional attitude report and in all of them, lexical markers mark either a narration or a result between the first segment and the matrix clause of the report. Finally, they also all seem to support anaphoric links between the reported content and the first segment. Both result and narration are thought to be coordinating relations. So even if we use the subordinating background between $\pi_{a}$ and $\pi_{b}$ in 5 , we cannot account for these links without violating RFC.

Examples in 7 thus allow us to see that the Intensional/Evidential treatment comes with the side effect of sometimes preventing from linking to the previous discourse. But

\footnotetext{
${ }^{9}$ Representing SDRS as directed acyclic graphs as it is often done is very confusing in this case, because a graph based representation does not distinguish which complex segment actually hosts such a cross-relation. The graphs for our structure and the problematic one are the same.
} 
they are very specific in the sense that they enforce an explicit rhetorical link from the previous discourse on the two level (the main discourse, and the embedded one) at the same time. The problem may however be more general if these links may as well be implicit.

Example 8. (1) [The factory blew up. $]_{a}[\text { John told me }]_{b}[$ there were a lot of dangerous chemicals in there. $]_{c}$

(2) [The factory blew up. $]_{a}$ [John thinks $]_{b}$ [there were a lot of dangerous chemicals in there. $]_{c}$

(3) [The factory blew up. $]_{a}[\text { John thinks }]_{b}$ [there were a lot of dangerous chemicals in there. $]_{c}[\text { But sam thinks }]_{d}$ [someone lighted a fire. $]_{e}$

Examples in 8 intend to illustrate this. The first one does not seem to require an implicit relation between $a$ and $c$. The possible explanation of the explosion by the presence of chemicals is not a mandatory part of what John said. Actually John might have said that to the writer even before the explosion happened, and the writer is making the link himself from what John previously said. The two other examples on the other hands may carry such implicit links between $a$ and the reported content $b$ : There is at least one plausible reading for the second example involving a coordinating relation between $a$ and $b$ which fits very well an implicit explanation between $a$ and $c$. The explosion actually made John think of a plausible explanation, which is that they are dangerous chemicals in the usine, and that these chemicals may have cause the explosion. Finally, the last example requires implicit explanation relations to make a better sense of the con trast relation that links $b$ and $c$. The beliefs of John and Sam are fully compatible, unless what John and Sam respectively said is explanation $(a, c)$ and explanation $(a, e)$, in which case they are not.

All together, this threatens to make SDRT better understanding of anaphoric links in attitude reports only come at the price of some wrong predictions in some intensional cases.

\section{$5 \quad$ Restoring accessibility}

We have shown that SDRT damages more standard but essentially correct accounts of anaphoric links going between modal and factual contexts. An account of attitude reports in DRT for instance, would not have this behaviour. Examples like 7 would introduce reference to events in the main DRS from the modal context, which is permitted. We would like such a behaviour, but with SDRT treatment of accessibility still applying inside the reported speech. To this end, we could drop the attribution relation, falling back to a DRT like treatment. The structure of one of our problematic report in SDRT would thus be sketched by $R_{\text {coord }}\left(\pi_{a}, \pi_{a t t}\right)$ with $\mathcal{F}\left(\pi_{a t t}\right)=K_{\pi_{b}} \wedge A(x, \phi) \wedge \phi \sim^{\wedge} \pi_{c}$. This structure allows referents in $\pi_{c}$ to attach or refer to elements in $\pi_{a} \cdot{ }^{10}$ This builds on intensional report being " closed" discursive structures. We showed in section 4 that a relation cannot really penetrate the report from the factual context without (a "copy" of) its left argument and itself being embedded under the attitude. Moreover, attachment to the matrix clause and attachment to a complex segment made of both the matrix clause and the report are semantically and dynamically equivalent. This allows us to abstract the complete speech act of reporting under a complex segment

\footnotetext{
${ }^{10}$ Such an approach actually needs to slightly modify the syntax of the SDRS language
} 
$\pi_{a t t}$. This approach however requires to adapt the language for inferring the relations because the intensional and evidential cases are now asymmetric. One has to state that the content of a segment $\pi_{m a t}$ in the evidential case is equal to a part of the content of the abstracted complex segment $\pi_{a t t}$ in the intensional case.

That is why we propose below a more conservative approach that makes use of continuation-syle semantics [4]. Continuation style semantics represents a discourse as a $\lambda$-abstraction of type $\llbracket \Gamma \rrbracket=\gamma \rightarrow((\gamma \rightarrow l \rightarrow t) \rightarrow l \rightarrow t)$ where $\gamma$ is the type of input contexts. A discourse thus asks for (i) an input context $i$ of type $\gamma$ containing the effects of processing the previous discourse; (ii) a continuation $o$ of type $\gamma \rightarrow l \rightarrow t$ representing the discourse to come and; (iii) a label $\pi$, the label of the SDRS representing the whole discourse.

To represent chunks of an SDRS, a language is used where every $n$-ary becomes an $n+1$-ary predicate, the extra argument stands for the label that hosts the predicate: A label $\pi$ with $\mathcal{F}(\pi)=R\left(\pi_{1}, \pi_{2}\right)$ will be represented as $\exists \pi_{1} \exists \pi_{2} \exists \pi R\left(\pi_{1}, \pi_{2}, \pi\right)$.

We will assume that the context contains a structural representation of the SDRS for the previous discourse such that the following functions may be defined:

(1) sel $_{l}: \gamma \rightarrow l$ that selects a label for attachment.

(2) $\nu: \gamma \rightarrow l \rightarrow \gamma$ that performs the SDRT update operation on the context [3], defined in terms of SDRT's language for inferring relations. Given a label $\pi$, it basically picks up a relation and two other labels $\pi_{1}, \pi_{2}$ in the context and add the relation $R\left(\pi_{1}, \pi, \pi_{2}\right)$ to the context.

Finally, we will use the following version of the binder rule to join a discourse and a sentence:

$$
\left.\llbracket D . S \rrbracket=\lambda i o \pi \exists \pi_{D} \llbracket D \rrbracket i\left(\lambda i^{\prime} \exists \pi_{S} \llbracket S \rrbracket i^{\prime} o \pi_{S}\right)\right)
$$

The main idea, is to refine [6] proposal of a lexical entry for attitude reports using continuation-style semantics to overcome the right-frontier problems. Since evidential and intensional readings only differ by the way the matrix clause and the embedded content are related, one simple solution is to postpone attachment of the matrix clause until the embedded content has been dealt with and all attachment to previous context have been done. But it must be performed before the following discourse is processed in order to still benefit from the intensional/evidential distinction. This might be done by modifying the continuation of the report in such a way that it proceeds to the attachment of the matrix clause before applying the real continuation.

Let us assume an attitude $\alpha$ in a discourse " $x \alpha$ that $\phi "$ and that syntax delivers us a parse leading to $\alpha(x, \phi)$. We add the lexical entry given in 2 for an attitude verbe $\alpha$, with $A$ a modal operator corresponding to attitude $\alpha$.

$$
\llbracket \alpha \rrbracket=\lambda x \lambda s \lambda i o \pi_{m a t t} \exists \phi A\left(x, \phi, \pi_{m a t t}\right) \wedge \exists \pi_{s} \phi \sim \pi_{s} \wedge s i\left[\lambda i^{\prime} o\left(\nu\left(\nu\left(i^{\prime}, \pi_{m a t t}\right), \pi_{s}\right)\right)\right] \pi_{s}
$$

Let us now have a look back to [The train arrived late $]_{a}$. [Then the company annouced that $]_{b}$ [the passengers should thus be refunded $]_{c}$.

We assume for $a$ a lexical entry like:

$$
\lambda i o \pi \exists x \operatorname{train}(x, \pi) \wedge \operatorname{Late}(x, \pi) \wedge o \nu(i, \pi)
$$


In this entry the update operation $\nu(i)$ will deliver a context $i^{\prime}$ containing the structure $\pi_{a} \mid F\left(\pi_{a}\right)=[x \mid \operatorname{train}(x) \wedge$ late $(x)]$, and maybe a relation linking $\pi_{a}$ to the previous context. Assuming the lexical entry for Thus is

$$
\llbracket \text { thus } \rrbracket=\lambda i o \pi s s i\left(\lambda i^{\prime} \operatorname{Result}\left(\operatorname{sel}_{L}\left(i^{\prime}\right), \pi, \operatorname{sel}_{L}\left(i^{\prime}\right)\right) \wedge o i^{\prime}\right)
$$

We end up with the following entry for the embeded content $c$ :

$$
\begin{aligned}
& \lambda i o \pi \exists y, z \wedge \text { The_Passengers }(y, \pi) \wedge \operatorname{Be} e_{-} \operatorname{Refunded}(y, \pi) \\
& \wedge \operatorname{Result}\left(\operatorname{sel}_{L}(i), \pi, \operatorname{sel}_{L}(i)\right) \wedge o i
\end{aligned}
$$

The lexical entry for to announce (our $\alpha$ here) will be given the_company as its first argument and the interpretation of $c$ as its second. Which should yield after beta reduction:

$$
\begin{aligned}
& \left.\lambda i o \pi_{\text {matt }} \exists \phi A \text { (The_company, } \phi, \pi_{\text {matt }}\right) \wedge \exists \pi_{s} \phi \sim \pi_{s} \\
& \wedge \exists y, z \wedge \text { The_Passengers }\left(y, \pi_{s}\right) \wedge \operatorname{Be} e_{-} \text {Refunded }\left(y, \pi_{s}\right) \\
& \wedge \operatorname{Result}\left(\operatorname{sel}_{L}(i), \pi_{s}, \operatorname{sel}_{L}(i)\right) \wedge o\left(\nu\left(\nu\left(i, \pi_{\text {matt }}\right), \pi_{s}\right)\right)
\end{aligned}
$$

When composing with $\llbracket a \rrbracket$, this entry will receive the context $i^{\prime}$ containing the structure $\pi_{a} \mid F\left(\pi_{a}\right)=[x \mid \operatorname{train}(x) \wedge$ late $(x)]$, unmodified, as input context and thus be able to select $\pi_{a}$ as first argument for the result relation without RFC violation. Importantly, successive call to the $\nu$ function will perform the intensional/evidential choice and choose a relation to link the report to the preceding discourse before processing the continuation.

\section{Conclusion}

Segmenting discourse structure cannot be avoided, but as we have shown, the discourse structure of segmented reports is not straightforward. We have thus given a more precise picture of what it should be and why. It remains to give a precise semantics to those reports, and especially to decide what is the content ${ }^{\wedge} \pi$ of a SDRS and what amount of structure it carries.

\section{References}

1. Asher, N.: A typology for attitude verbs and their anaphoric properties. Linguistics and Philosophy 10(2), 125-197 (1987)

2. Asher, N., Hardt, D., Busquets, J., Sabatier, I.P.: Discourse parallelism, ellipsis, and ambiguity. Journal of Semantics 18, 200-1 (2001)

3. Asher, N., Lascarides, A.: Logics of Conversation (Studies in Natural Language Processing). Cambridge University Press (Jun 2005), http://www .worldcat.org/ isbn/0521659515

4. Asher, N., Pogodalla, S.: SDRT and Continuation Semantics. In: Onada, T., Bekki, D., McCready, E. (eds.) New Frontiers in Artificial Intelligence JSAI-isAI 2010 Workshops, LENLS, JURISIN, AMBN, ISS, Tokyo, Japan, November 18-19, 2010, Revised Selected Papers, LNCS, vol. 6797, pp. 3-15. Springer (2011), http://hal. inria.fr/inria-00565744

5. Danlos, L., Rambow, O.: Discourse relations and propositional attitudes. Constraint in Discourse (CID) (2011) 
6. Hunter, J., Asher, N., Reese, B., Denis, P.: Evidentiality and intensionality: Two uses of reportative constructions in discourse. In: Proceedings of the Workshop on Constraints in Discourse. pp. 99-106. Maynooth: National University of Ireland (2006)

7. Kamp, H., Reyle, U.: From Discourse to Logic: Introduction to Model-theoretic Semantics of Natural Language, Formal Logic and Discourse Representation Theory, Studies in Linguistics and Philosophy, vol. 42. Kluwer, Dordrecht (1993)

8. Kaplan, D.: Quantifying in. Synthese 19(1-2), 178-214 (1968)

9. Maier, E.: Presupposing acquaintance: a unified semantics for de dicto, de re and de se belief reports. Linguistics and Philosophy 32(5) (2010), http://www. springerlink.com/index/10.1007/s10988-010-9065-2

10. Vieu, L.: On the semantics of discourse relations. Constraint in Discourse (CID) (2011) 